\title{
Digital Craftsmanship in the Wearable Senses Lab
}

\author{
Kristina Andersen \\ Industrial Design \\ Eindhoven University of \\ Technology (TU/e) \\ Eindhoven Netherlands \\ h.k.g.andersen@tue.nl
}

\author{
Marina Toeters \\ design \& research in fashion tech \\ by-wire.net / Eindhoven \\ University of Technology (TU/e) \\ Utrecht / Eindhoven Netherlands \\ marina@by-wire.net
}

\author{
Bruna Goveia \\ Industrial Design \\ Eindhoven University of \\ Technology (TU/e) \\ Eindhoven Netherlands \\ b.goveia.da.rocha@tue.nl
}

\author{
Angella Mackey \\ Industrial Design \\ Eindhoven University of \\ Technology (TU/e) \\ Eindhoven Netherlands \\ a.m.mackey@tue.nl
}

\author{
Oscar Tomico \\ Industrial Design \\ Elisava / TU/e \\ Barcelona / Eindhoven \\ Spain / Netherlands \\ otomico@elisava.net
Troy Nachtigall
Industrial Design
Technology (TU/e)
Eindhoven Netherlands
t.r.nachtigall@tue.nl \\ Eindhoven University of
}

\begin{abstract}
Wearable and e-textiles as a field has tended to overlook its own documentation, as notions and overarching ideas are developed over time and across individual projects. We would like to begin addressing this by charting the development of Digital Craftsmanship through a number of projects over time. Practically, we propose to show a small selection of garments and samples alongside a simple framework for future documentation of work.
\end{abstract}

\section{CCS CONCEPTS}

H.5.m. Information interfaces and presentation (e.g., HCI): Miscellaneous

\section{KEYWORDS}

Digital craftsmanship; documentation, theory development, soft wearables; interaction design; dynamic fabric.

\section{ACM Reference format:}

Kristina Andersen, Bruna Goveia, Oscar Tomico, Marina Toeters, Angella Mackey, Troy Nachtigall. 2019. Digital Craftsmanship in the Wearable Senses Lab. ISWC '19, September 9-13, 2019, London, United Kingdom $\odot 2019$ Association for Computing Machinery. ACM, 6 pages. https://doi.org/10.1145/3341163.3346943

\section{Introduction}

The field of wearable soft things or soft wearables has developed from a niche concern to an increasingly broad area of research $[5,9,11,19]$. As high performing materials have become more widely available and our systems of making and production

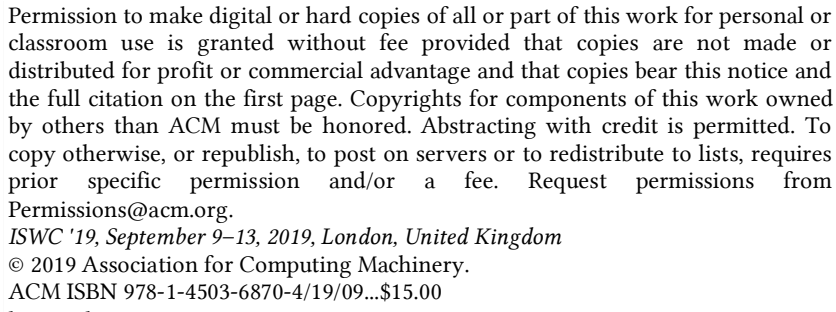
classroom use is granted without fee provided that copies are not made or distributed for profit or commercial advantage and that copies bear this notice and the full citation on the first page. Copyrights for components of this work owned by others than ACM must be honored. Abstracting with credit is permitted. To copy otherwise, or republish, to post on servers or to redistribute to lists, requires prior specific permission and/or a fee. Request permissions from Permissions@acm.org.

ISWC '19, September 9-13, 2019, London, United Kingdom

(c) 2019 Association for Computing Machinery.

ACM ISBN 978-1-4503-6870-4/19/09...\$15.00

more sophisticated, we see wearable electronics projects emerge not only from the arts and fields of technology, but also from fashion, design and engineering. With the so-called 4th industrial revolution promising a much more flexible and automated factory work floor, we may soon see increasing levels of simple traditional electronics incorporated into soft things in our everyday lives [4].

We believe that the future of soft wearables is now broadening up to include programming, not just electronics and interactive behaviour, but programming the whole garment in terms of its material, its form, its manufacture, its level of personalisation, associated services, and its direct relation to both its user and the social cultural and economic structures around it. In order for such complex frameworks to emerge, we need structures and terminologies not just to analyse past work but to frame new projects and developments as they emerge.

\section{Digital Craftsmanship}

Over the last ten years, the notion of digital craftsmanship has developed through the building of our research projects and teaching in the lab. The term emerged as an attempt to describe the skill of expressing a designerly or artistic aim, manifested through soft physical materials, through a system underpinned by the digital material.

In our understanding, this digital material stretches from technological inspiration, technology envisioned in a project, technology required for execution, to technology for manufacture. Instead of considering the physical execution of an idea as the last and final step of a process, we wanted to allow material exploration, physical craftsmanship and skill to be central to our work with a set of materials that increasingly include digital material and notions. 
This means that our projects increasingly combine programming, mathematics and material explorations, making use of crafting and open-ended making to imagine and craft new digital/physical objects. The digital often constitute the core material to be expressed through embodied objects ranging from the mundane to the complex; cardboard, recycling, fabric, embroidery, metal, printing and laser cutting etc. As a result, we are essentially working on material explorations of immaterial ideas in the context of post-human technology and new materialism $[1,14,16]$.

In practice, this means that we consider digital assets design material, to be addressed and worked with in craft-like techniques, through hands-on making and sketching, moving through a number of computational design and manufacturing techniques all aimed at producing explorative designs of material/digital objects. All this manifest through a very handson craft approach to working with machinery such as flexible substrate 3D printers, laser cutters (fabric, vinyl, leather etc.), digital embroidery weaving and knitting. In this, our aim is to explicate our understandings of both machines and making towards a point, where we can make use of the technologies available to us as expressive mediums for craftsmanship.

\section{Documentation Framework}

To this end, we have focused on understanding and analysing our own lab outcomes, what they explored, how they were expressed, and the extent to which they requiring a renewed understanding of the industrial work floor and its possibilities, as we work towards developing a renewed understanding of the roles of machines and aesthetics. These projects have already been documented in a number of publications on individual project level $[2,7,8,10,12,13,17]$, but here we wanted to expand the scale, to be able to begin looking across projects over time.

In order to do this, we developed a simple framework, seen in fig. 1. By looking through these projects we see three levels of complexity in the manner in which they each relate to the data in and around their own production and designs. The three levels are the product level, the system level and the service level.

1. At a product level (digital material production), the development of wearable technology is driven in part by the culture of "maker" DIY digital fabrication. Digital knitting, weaving and printing allow us to combine yarns, fibres or filaments with different material properties in different ways (structures) for each part of a garment. This is widening the design and production space, but also allows us to consider the craft aspects of innovative textile work.

2. At a system level (personalized design process), algorithmic treatment of digital personal data allows the shape, design and finishing to be programmable and therefore modifiable in a way that still keeps the identity of the designer while the fitting to the customer's identity, moving body, and context of use. In the following examples, we see increasing levels of digital complexity on the designs.

3. At a service level (end user programming), we can use digital sensing and actuating capabilities to enhance its physical properties by adding control systems integrated in the textile. In this way "Self-programmed" behaviour and functionality allow us to create new applications by programming the mapping between digital functionality and physical interaction. In the following examples, the complexity present in the designs continues to increase as the wearable is used and experienced by its owner.

As a crucial part of sharing and understanding this process we believe that making the physical material available alongside the analysis of their context is a key component is allowing digital design and craftsmanship to develop a research culture for the future $[3,6,15,18]$.

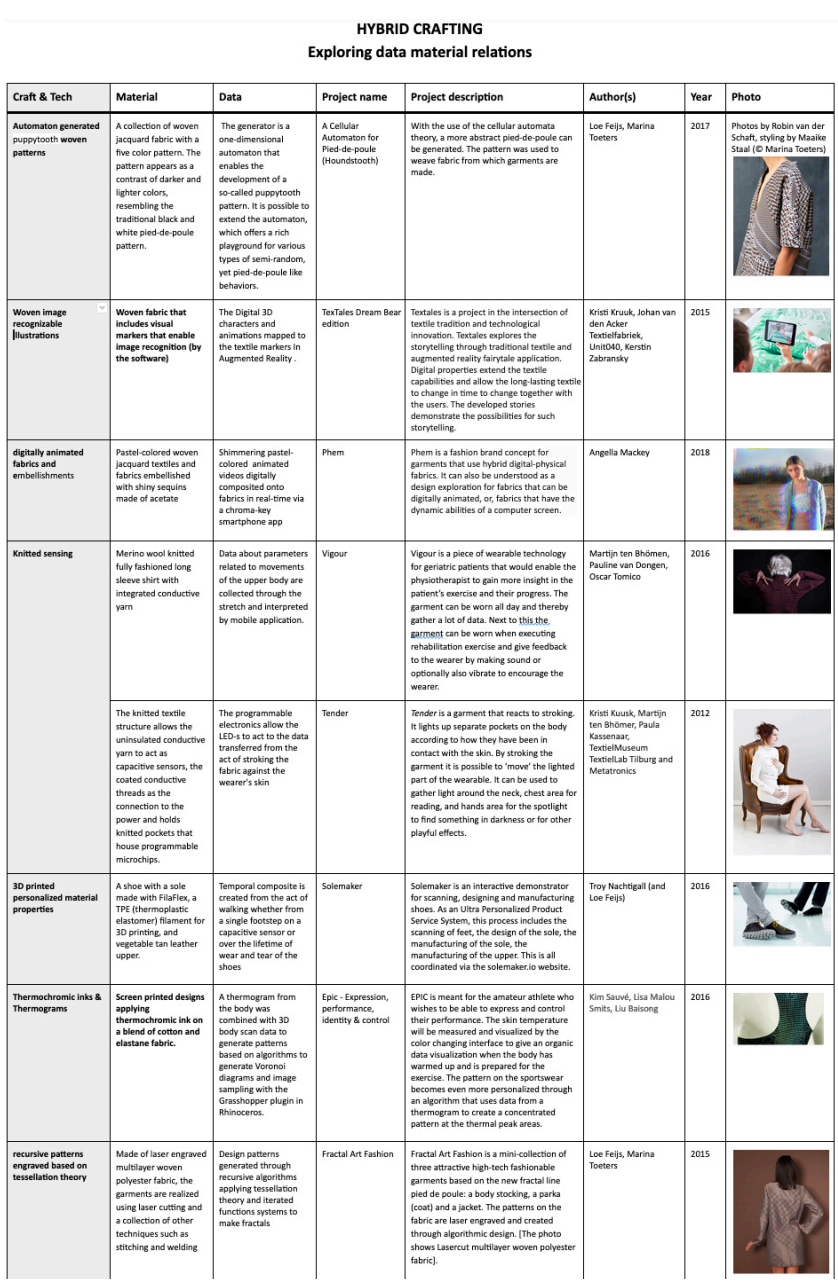

Figure 1: Part of an example page of documentation framework. 


\section{Examples of Work}

In the following figures (figure 2-5) we will show a number of project examples. We propose a selection of them for the exhibition.

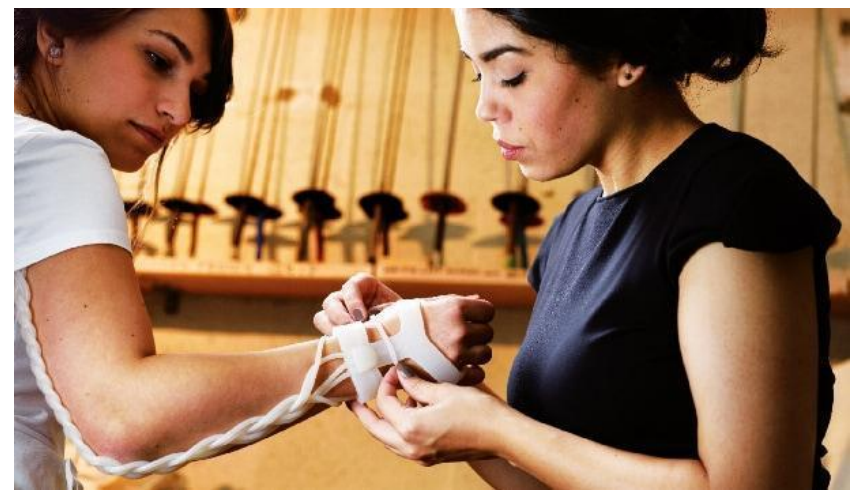

Figure 2: Flow (2015). Bruna Goveia da Rocha. Flow is a wearable that includes six inflatable chambers that push against the body to cue fundamental joint movements of the wrist and forearm to foster learning physical activities, such as fencing. The integrated chambers and air ways in Flow operate as a material extension of the actuators responsible for the inflation; removing the air pumps from the area of actuation. [10]

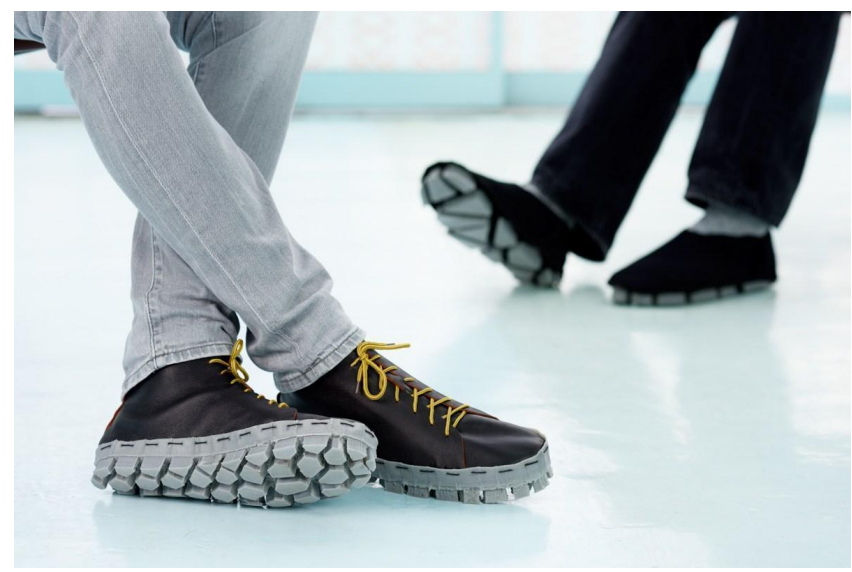

Figure 3: Solemaker (2016). Troy Nachtigall (and Loe Feijs). Solemaker is an interactive demonstrator for scanning, designing and manufacturing shoes. As an Ultra Personalized Product Service System, this process includes the scanning of feet, the design of the sole, the manufacturing of the sole, the manufacturing of the Upper. [14]

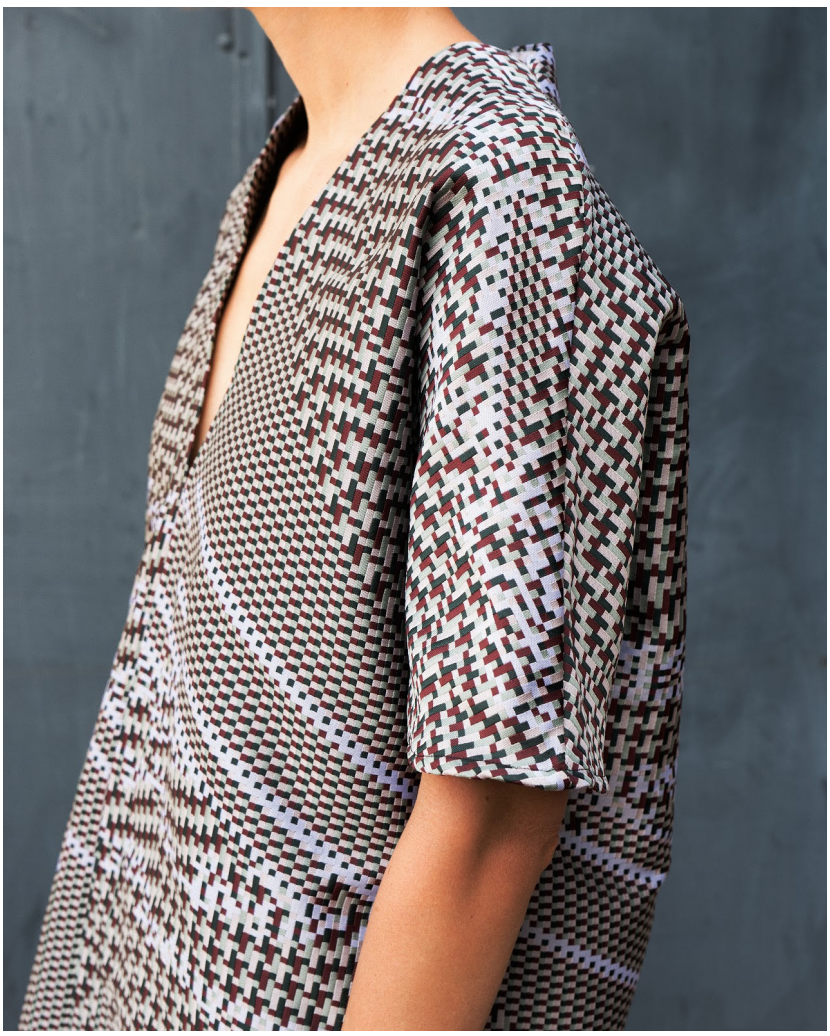

Figure 4: A Cellular Automaton for Pied-de-poule (Houndstooth) (2017). Loe Feijs, Marina Toeters. With the use of the cellular automata theory, a more abstract piedde-poule can be generated. The pattern was used to weave fabric from which garments are made. [8] Photo by Robin van der Schaft, styling by Maaike Staal (๑ Marina Toeters)

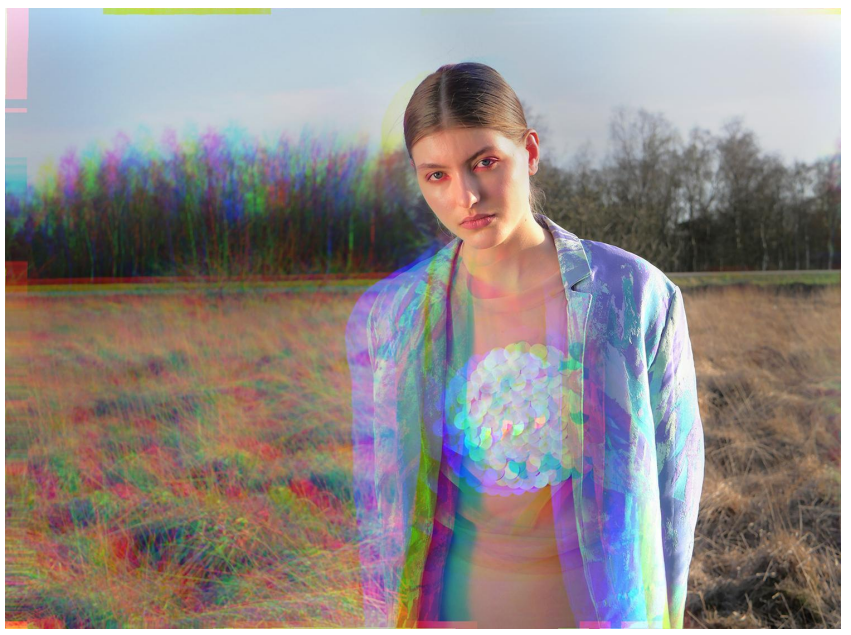

Figure 5: Phem (2018). Angella Mackey. Phem is a fashion brand concept for garments that use hybrid digitalphysical fabrics. It can also be understood as a design exploration for fabrics that can be digitally animated, or, fabrics that have the dynamic abilities of a computer screen. [12] 


\section{Proposal}

For ISWC, we would like to propose an exhibit of garments and samples originating from our lab over a longer period of time. We will pick three examples, one from each of the categories mentioned above. These examples are all exhibition ready, but we will make the final selection when we have a better understanding of the space of the exhibition. Together these samples will illustrate and document past work at the lab, but also provide evidence of the process of developing the notion of digital craftsmanship and how we use this term and practice in the lab today.

\section{Requirements for Exhibit}

Our garments and samples have no technical requirements, in the sense that they are stand alone. If possible, we would like to present at least one on a mannequin, but we can also have them on hangers or lying flat on a table. In addition to this we would like to distribute our data sheet as seen in fig. 1. as a proposal for the beginning of a future taxonomy of digital craftsmanship work.

\section{Vision for the Future Past}

We are hoping that through showing not just examples of work at ISWC but also our simple framework for analysis, we can engage the community in conversation about not just what we did, but also what we may do next, how if fits with other work and communities and how we may document and share out experiences and knowledge.

\section{Conclusion}

We believe that future of wearable soft things may still have technology physical embedded, but they may also be nontechnical devices, where the technological innovation lies in the manner of their manufacture, the way they are personalized towards their users and uses, and finally in how they are related to product service systems throughout their use cycle. In order to build such visions of the future we want to refine and evolve the notion of digital craftsmanship. Exhibiting at ISWC is a step towards doing this in conversation and collaboration with our wider wearable community.

\section{ACKNOWLEDGMENTS}

We would like to specially thank the authors of the works and all the staff, students and guests that have been collaborating with the lab over the years.

\section{REFERENCES}

[1] Andersen, K., 2017: Making Speculative Technologies, in Intersecting Art and Technology in Practice: Techne/Technique/Technology, Routledge, pp. 42-49. ISBN-13: 978-1138934115

[2] Andersen, K. and Knees, P., 2016. The Dial: Exploring Computational Strangeness in proc. of CHI 2016, ACM, pp. 1352-1358. doi $>10.1145 / 2851581.2892439$
[3] Andersen, K., Boucher A., Chatting D., Desjardin A., Devendorf L., Gaver, W., Jenkins, T., Odom, W., Pierce, J. and Vallgårda, A., 2019, Doing Things with Research thru Design: With What, with Whom, and Towards What Ends? CHI2019.

[4] Andersen, Kristina, and Joanna Berzowska. 2006. "Worn Technology: Altering Social Spaces". Open, Hybrid Space 11: 148-157.

[5] Burtch, G., Yoo, Y., \& Weiss, A. (2010, December). Digital Innovation and Craftsmanship: the Case of CF Martin \& Company. In ICIS (p. 152).

[6] Devendorf, L., Andersen, K., Wakkary, R. Rosner, D. K., and Pierce, J., 2019. From HCI to HCI-Amusement: Strategies for Engaging what New Technology Makes Old. CHI2019.

[7] Feijs, Loe, and Marina Toeters, (2015). A novel line fractal pied de poule (houndstooth). In Proceedings of the Conference of Bridges: Mathematics, Music, Art, Architecture, Culture (pp. 223-230).

[8] Feijs, Loe, and Marina Toeters, 2017. "A cellular automaton for pied-de-poule (houndstooth)". Proceedings of Bridges 2015: Mathematics, Music, Art, Architecture, Culture: 403-406. Phoenix: Tessellations Publishing.

[9] Frankjær, R., \& Dalsgaard, P. (2018, June). Understanding Craft-Based Inquiry in HCI. In Proceedings of the 2018 Designing Interactive Systems Conference (pp. 473-484). ACM.

[10] Goveia, Bruna and Oscar Tomico. 2019. "Flow: Towards Communicating Directional Cues through Inflatables". Extended Abstracts of the $2019 \mathrm{CHI}$ Conference on Human Factors in Computing Systems (LBW1313). ACM, New York, NY, USA. DOI: https://doi.org/10.1145/3290607.3312828

[11] Jacobs, J., Mellis, D., Zoran, A., Torres, C., Brandt, J., \& Tanenbaum, J. (2016, June). Digital craftsmanship: HCI takes on technology as an expressive medium. In Proceedings of the 2016 ACM Conference Companion Publication on Designing Interactive Systems (pp. 57-60). ACM.

[12] Mackey, Angella, Stephan Wensveen, Ron Wakkary, Annika Hupfeld, Oscar Tomico. 2019. "Wearing Digital Shimmers: A fashion-centric approach to wearable technology". Proceedings of the 4th Biennial Research Through Design Conference, 19-22 March 2019, Delft and Rotterdam, The Netherlands, DOI: https://doi.org/10.6084/m9.figshare.7855862.v1

[13] Nachtigall, Troy, and Kristina Andersen. 2018. "Making Secret Pockets". Extended Abstracts of the $2018 \mathrm{CHI}$ Conference on Human Factors in Computing Systems (LBW574). ACM, New York, NY, USA

[14] Nachtigall, Troy, Oscar Tomico, Ron Wakkary, and Pauline van Dongen. 2019. "Encoding Materials and Data for Iterative Personalization". Proceedings of the 2019 CHI Conference on Human Factors in Computing Systems. ACM, New York, NY, USA https://doi.org/https://doi.org/10.1145/3290605.3300749

[15] Odom, W., Jenkins, T., Andersen, K., Gaver, B., Pierce, J., Vallgårda, A., ... \& Lefeuvre, K. (2018). Crafting a place for attending to the things of design at CHI. interactions, 25(1), 52-57.

[16] Philp Ross and Stephan Wensveen. 2010. Designing aesthetics of behavior in interaction: Using aesthetic experience as a mechanism for design. International Journal of Design, 4(2), 3- 13 http://www.ijdesign.org/index.php/IJDesign/article/viewFil e/765/294

[17] ten Bhömer, Martijn, Oscar Tomico, Stephan Wensveen. 2015. "Designing ultra-personalized embodied smart textile services for well-being”. Advances in Smart Medical Textiles: Treatments and Health Monitoring: 155-175. Cambridge: Woodhead Publishing.

[18] Tomico, Oscar, Danielle Wilde. 2016. "Soft, embodied, situated \& connected: enriching interactions with soft wearables". mUX: The Journal of Mobile User Experience, 5 (1).

[19] Tomico, Oscar, Lars Hallnäs, Rong-Hao Liang, Stephan Wensveen. 2017. "Towards a next wave of wearable and fashionable interactions". International Journal of Design 11 (3): 1-6. 